\title{
NOISE AND OSCILLATIONS IN GOLD-DOPED GERMANIUM PHOTODIODES
}

\author{
P. T. BOLWIJN, C. v.d. RIJST, W. G. vAN AST and T. LAM \\ Physical Laboratory, State University, Utrecht, The Netherlands
}

(Received 6 June 1966)

\begin{abstract}
Considerable noise effects in excess of shot noise and oscillations found in commercially available, gold-doped germanium photodiodes have been investigated. The noise and oscillation effects occur in the photocurrent of reversely biased diodes at temperatures below about $100^{\circ} \mathrm{K}$. The dependence of the observed phenomena on external parameters, such as voltage, current, illumination level and temperature has been studied. The excess noise is attributed to a modulation of the voltage across the junction due to generation-recombination fluctuations of the large bulkresistance. The voltage across the junction modulates the current through the device, since the differential resistance of the junction is not infinite. There is reasonable agreement between the experiments and a simple theoretical model. The oscillations, which are not directly connected with the excess noise, are believed to arise owing to the presence of the gold levels, and might be associated with the recombination instability described by Bonch-Bruevich and Kalashnikov. In order to arrive at more definite conclusions better defined structures should be investigated.
\end{abstract}

Résumé-Les effets considérables de bruit en excédent du bruit shot et des oscillations obtenues dans les photodiodes en germanium à dope d'or en usage commercial ont été examinés. Les effets de bruit et d'oscillation se produisent dans le courant photoélectrique des diodes à polarisation inverse à des températures inférieures à environ $100^{\circ} \mathrm{K}$. La dépendance des phénomènes observés en fonction des paramètres externes tels que la tension, le courant, le niveau d'illumination et la température a été étudiée. Le bruit excédent est attribué à une modulation de la tension entre la jonction causée par les fluctuations génération-recombinaison de la haute tension de masse. La tension entre la jonction module le courant à travers le dispositif puisque la résistance différentielle de la jonction n'cst pas infinic. Il cxiste un bon accord entrc les cxpériences ct le modèle théorique simple. Les oscillations qui ne sont pas directement associées avec le bruit excédent sont probablement produites par la présence de niveaux d'or et peuvent être associées à l'instabilité de recombinaison décrite par Bonch-Bruevich et Kalashnikov. Pour arriver à des conclusions plus exactes, de meilleures structures définies devraient être examinées.

Zusammenfassung-Bcträchtliche Rauscheffcktc, dic in kommerziellen golddotierten Germaniumfotodioden zusätzlich zum Schrotrauschen und Oszillationen auftreten, wurden untersucht. Das Rauschen und Oszillationen in Fotostrom von rückwärts gepolten Dioden kommen bei Temperaturen unterhalb etwa $100^{\circ} \mathrm{K}$ vor. Die Abhängigkeit der beobachteten Erscheinungen von äusseren Parametern wie Spannung, Strom, Beleuchtungsstärke und Temperatur wurden aufgenommen. Das zusätzliche Rauschen wird einer Modulation der Spannung am $p n$-Übergang zugeschrieben, welche infolge Fluktuationen der Generations- und Rekombinationsprozesse und der damit verbundenen Widerstandsänderungen zustande kommen. Die Spannung am pnÜbergang moduliert den Strom, weil der differentielle Widerstand nicht unendlich gross ist. Zwischen Experimenten und einem einfachen theoretischen Modell besteht hinreichende Úbereinstimmung. Die Oszillationen, welche keine direkte Verbindung mit dem zusätzlichen Rauschen zeigen, verdanken ihre Entstehung vermutlich dem Vorhandensein der Goldniveaus und können vielleicht mit der von Bonch-Bruevich und Kalashnikov beschriebenen Rekombinationsinstabilität in Zusammenhang gebracht werden. Um genauere Schlussfolgerungen ziehen zu können sollten einfachere Strukturen untersucht werden. 


\section{NOTATION}

$\begin{array}{ll}N_{c} & \text { donor concentration } \\ N_{t} & \text { trap concentration } \\ n_{i} & \text { intrinsic carrier concentration } \\ n_{0}, p_{0} & \text { equilibrium concentration of free electrons and } \\ & \text { holes } \\ n_{1}, p_{1} & \begin{array}{l}\text { concentration of free electrons and holes when } \\ \text { the Fermi level coincides with the trapping level }\end{array} \\ \tau_{n}, \tau_{p} & \text { lifetime of electrons and holes } \\ \tau_{p_{0}} & \text { lifetime for holes in highly } n \text {-type material } \\ \tau_{r_{0}} & \text { lifetime for electrons in highly } p \text {-type material } \\ \sigma_{n}, \sigma_{p} & \text { electron and hole capture cross-section }\end{array}$

\section{INTRODUCTION}

Is aN investigation of the noise behaviour of $p-n$ junction photodiodes at low temperatures we observed noise in excess of shot noise as well as oscillations in the photocurrent in reversely biased, commercially available, gold-doped Ge diodes.

The noise in the current of reversely biased diodes generally satisfies the well-known shot noise formula. (1) Some additional low-frequency noise of the $1 / f$ type may be present due to surface states or due to contact noise. For low-injection levels the noise may be less than full shot noise because of traps in the transition region. ${ }^{(1)}$ Noise in excess of shot noise may be associated with avalanche noise or micro plasma fluctuations. ${ }^{(2-4)} \mathrm{HYDE}^{(5)}$ has found noise above shot noise with spectra of the form const. $/\left(1+\omega^{2} \tau^{2}\right)$ in diodes under reverse bias conditions at room temperature. The source of the noise remained unknown. The strong increase above the shot noise level we observed appears in the photocurrent below a critical temperature of about $100^{\circ} \mathrm{K}$. The noise density falls at frequencies near $100 \mathrm{kc} / \mathrm{s}$. This noise could be attributed to a modulation of the voltage across the junction due to generation- recombination noise of the high resistivity bulk material of the diode. The voltage across the junction modulated the current through the device, since the differential resistance of the junction was found to be finite.

Below the critical temperature we observed also sinusoidal oscillations in the photocurrent. Oscillation frequencies were in the range from $1 \mathrm{kc} / \mathrm{s}$ up to $1 \mathrm{Mc} / \mathrm{s}$. Instabilities in semiconductors have been described by several authors. ${ }^{(6-18)}$ Many of these instabilities occur only when a magnetic field is applied ${ }^{(6)}$ or are related to the well-known Gunn-effect or are due to transit-time effects. ${ }^{(7)}$ The frequencies of these oscillations are usually in $\mu_{n}, \mu_{p}$ mobility of electrons and holes

$L_{n}, L_{p}$ diffusion length of electrons and holes

$D_{n}, D_{p}$ diffusion coefficient of electrons and holes

$v \quad$ velocity of the cartiers

$E_{F} \quad$ energy of Fermi level

$E_{t} \quad$ energy of trapping level

$V$ voltage across the junction

$I$ current through the device

$R$ bulk resistance

$R_{\mathrm{i}} \quad$ differential resistance of the junction

the microwave region. The oscillations in the frequency range below about $10 \mathrm{Mc} / \mathrm{s}$, which are of interest to us, are associated with several effects. A negative differential conductivity due to doubleinjection, ${ }^{(8,9)}$ or due to field dependence of capture rate may play a part. ${ }^{(10.11)}$ Furthermore impurity breakdown ${ }^{(12)}$ and piezo-electric effects ${ }^{(13)}$ may give rise to instabilities. In many cases the origin of the oscillations is unknown or ascribed to contact properties. ${ }^{(14-18)}$

We investigated in detail the dependence of the observed oscillations on many external parameters. The information gained from these measurements was limited because of the complexity of our device. We believe that the oscillations arise in the bulk of the gold-doped $p$-region of the diode, and that the gold levels play an essential part. The oscillations might be related to the phenomena described by BoNCH-Bruevich and Kalashnikov. ${ }^{(19,20)}$

In Section 2 a short description of the device and the experimental techniques are given. The experimental results are described in Section 3 . Some attention was given to measurements above the critical temperature from which information could be gained on the presence of gold impurities and their concentration. The behaviour of photocurrent, noise and oscillations is described in detail as well as the search for the influence of ambient gas or surface treatment on the effects. In Section 4 we show the evidence of the presence of gold impurities, analyse the noise behaviour, and discuss the properties of the oscillation phenomena.

\section{DEVICE AND EXPERIMENTAL TECHNIQUES}

The diodes investigated are commercially available germanium photodiodes, obtained from Standard Telephones and Cables Limited, type 
$P G 50 A$. On request we have received the following information from the manufacturer*: In $n$-type, As doped, material of resistivity between 5 and $15 \Omega$-cm, a $p$-region is obtained by gold doping giving a junction $60 \mu$ from the end of the bar. Information on the gold concentration was not available. At the other end a $n^{+}$layer of approximately $22 \mu$ is obtained by diffusion of gold/antimony. The contacts are first nickelcoated, then golded and soldered. The dimensions of the face of the diode are $1.5 \times 0.5 \mathrm{~mm}^{2}$, and the depth of the diode is $0.75 \mathrm{~mm}$. The photosensitive area was given as $1.5 \times 0.1 \mathrm{~mm}^{2}$ and the cut-off wavelength as $1.8 \mu$. The maximum reverse voltage permitted is $100 \mathrm{~V}$. The reverse dark current at room temperature is smaller than $250 \mu \mathrm{A}$. The diodes are delivered encapsulated in a metal niount with a glass window. We mounted the diodes in a copper box which could be partly immersed in liquid nitrogen. The temperature of the sample was varied by controlling the height of the nitrogen level. The temperature of the diode was measured with a constantan-manganine thermocouple attached to the metal mount of the diode. During the measurements the temperature could be kept constant within $0.5^{\circ} \mathrm{K}$. The copper box contained a glass window, which made it possible to irradiate the diode during cooling. A normal incandescent lamp was used to irradiate the diode. In addition, the ambient gas of the diode can be varied by means of a gas inlet. In such experiments the glass window was removed from the metal mount. The effects to be described in this paper were also found when the diode was immersed directly in liquid nitrogen, both with and without mount. In some experiments the diode was etched in $15 \% \mathrm{H}_{2} \mathrm{O}_{2}$ at $70^{\circ} \mathrm{C}$. After etching the diode was washed in pure alcohol, and cleaned with dry nitrogen.

The noise measurements were performed in the frequency range from $1 \mathrm{kc} / \mathrm{s}$ to $1 \mathrm{Mc} / \mathrm{s}$ by means of a heterodyne noise analyser developed at our laboratory by VAN BURIK and WESSELS. ${ }^{(21)}$ In most cases the load resistance of the $p-n$ diode was $20 \mathrm{k} \Omega$. For calibration purposes a noise diode of the type K81A was used, showing full shot noise in the frequency range under investigation. The

\footnotetext{
* We are indebted to the Standard Telephones and
} Cables Limited for giving us this information. spectral noise densities $S_{i}(f)$ were expressed in terms of equivalent saturated diode current $I_{e q}$ defined by $S_{i}(f)=2 e I_{e q}$. The oscillations were analysed with the above mentioned analyser or measured directly with an a.c. meter and oscilloscope.

\section{EXPERIMENTAL RESULTS}

We investigated 16 samples. An increase of noise as well as oscillations were found in the reverse photocurrent at low temperatures between 77 and $100^{\circ} \mathrm{K}$ in 11 samples. One sample exhibited an increase of noise only and one sample exhibited oscillations only. There was also one sample in which the oscillation and enhanced noise were quenched by u.v. radiation. The last one will be dealt with in the Appendix.

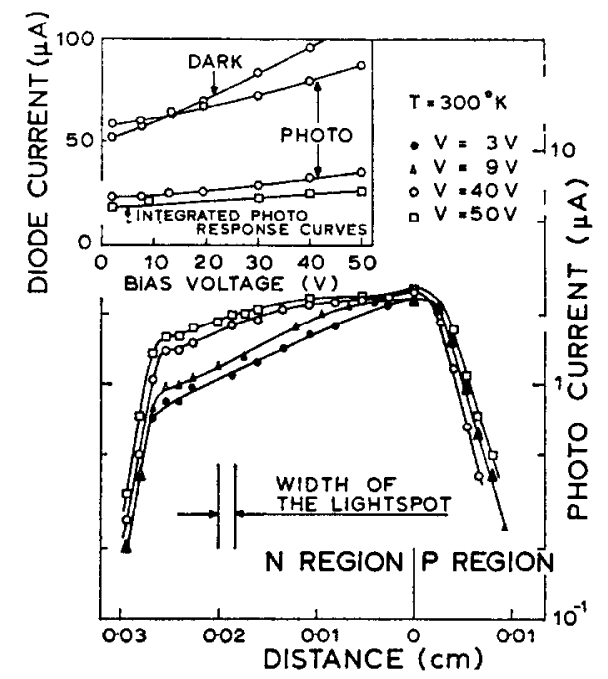

FIG. 1. Local photoresponse curves for various voltages and $I-V$ characteristics at room temperature.

\section{(a) Behaviour above the critical temperature}

The I-V characteristics of the reversely biased diode show a small linear increase of current with increasing voltage at room temperature, corresponding to a differential resistance of the order of magnitude of 1-10 M 2 . Light-spot measurements performed after the method of GouchER ${ }^{(22)}$ with a light strip $15-\mu$ wide, revealed a photosensitive area of $1.5 \times 0.3 \mathrm{~mm}^{2}$. Typical examples of $\mathrm{I}-\mathrm{V}$ characteristics and photoresponse curves are shown in Fig. 1. The increase of sensitive area with increasing voltage can be related to the increase of 
photocurrent at integral illumination (see the inset of Fig. 1). Unsaturated I-V characteristics may be caused by an inversion layer on the diode ${ }^{(23)}$ and by a drift current contribution to the total current. The latter possibility is the most likely, in our case, since the I-V characteristics, as shown in Fig. 1, were not affected after the surface had been etched. Note, in addition, that the resistivity of the $n$-region of the diode is rather high. A more detailed investigation regarding this point will be given elsewhere. ${ }^{(24)}$

The spectral noise density of the noise in the reverse current was found to be shot noise in the frequency range from $1 \mathrm{kc} / \mathrm{s}$ to $1 \mathrm{Mc} / \mathrm{s}$. At lower frequencies the noise appeared to be $1 / f$ noise. The shot noise formula is obeyed for voltages up to $100 \mathrm{~V}$, which implies that the voltage-dependent part of the reverse current is not caused by leakage currents. ${ }^{(24,25)}$ Some diodes showed noise densities which were somewhat lower than full shot noise, which might be caused by partly filled traps in the junction space charge region. (1)

When the temperature was lowered no surprising temperature dependence occurred down to a certain temperature, which was characteristic for the sample under investigation. This critical temperature lies between 90 and $110^{\circ} \mathrm{K}$.

The dark current decreases with decreasing temperature according to an exponential law. The activation energy found decreases from $\Delta E=0.67 \pm 0.02 \mathrm{eV}$ above $320^{\circ} \mathrm{K}$ to $\Delta E=0.50 \pm 0.02 \mathrm{eV}$ below $250^{\circ} \mathrm{K}$ at voltages below $1 \mathrm{~V}$. In the temperature range below $250^{\circ} \mathrm{K}$ the activation energy decreases with increasing voltage above $1 \mathrm{~V}$. The photocurrent decreases slightly with decreasing temperature. The dependence of the photocurrent on temperature is not easy to predict, since there are several competing factors, which contribute to this dependence (e.g. variation in bandgap, minority carrier lifetime and diffusion coefficient with temperature).

The I-V characteristics do not change essentially with temperature; only the linear increase of current with increasing voltage becomes somewhat smaller at low temperatures. The noise density remains at or near the shot noise level in the temperature range down to about $100^{\circ} \mathrm{K}$.

\section{(b) Behaviour below the critical temperature}

Below the critical temperature the I-V

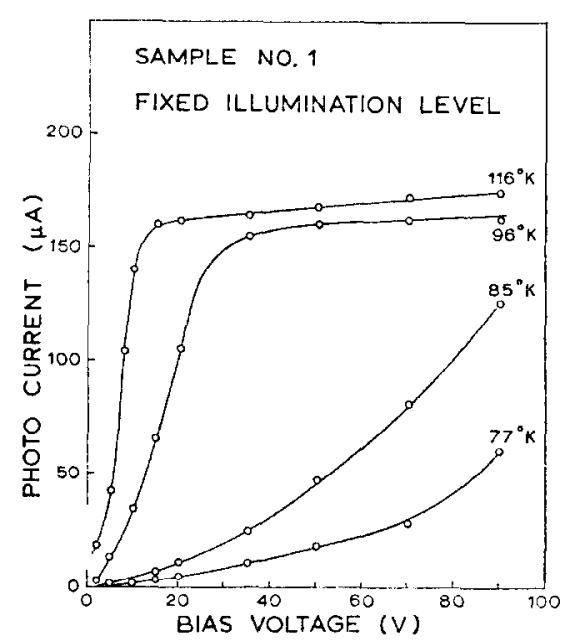

FIG. 2. $T-V$ characteristics at high fixed illumination level and at various temperatures.

characteristics change, the noise in the photocurrent increases strongly, and one or more coherent oscillations appear in the photocurrent. We will now describe the dependence of these quantities on several parameters, such as voltage, current, illumination level and temperature.

(i) Photocurrent. A typical example of the static $\mathrm{I}-\mathrm{V}$ characteristics is shown in Fig. 2 at a high fixed illumination level and for different temperatures. The increase of the current with voltage at the highest voltages and at the lowest temperatures was sometimes stronger than shown in Fig. 2, but it could then be removed by etching of the diode. Figure 3 shows a typical example of the characteristics at lower illumination levels. The temperature in this Figure was fixed and the

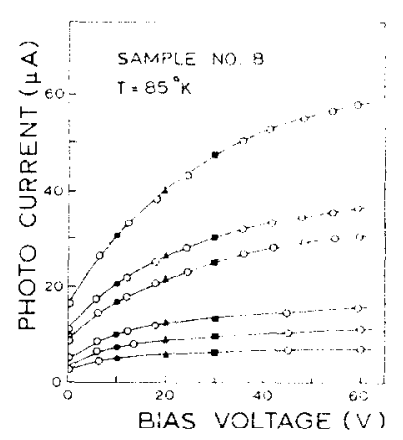

FIG. 3. I-V characteristics at fixed temperature and for various low illumination levels. 


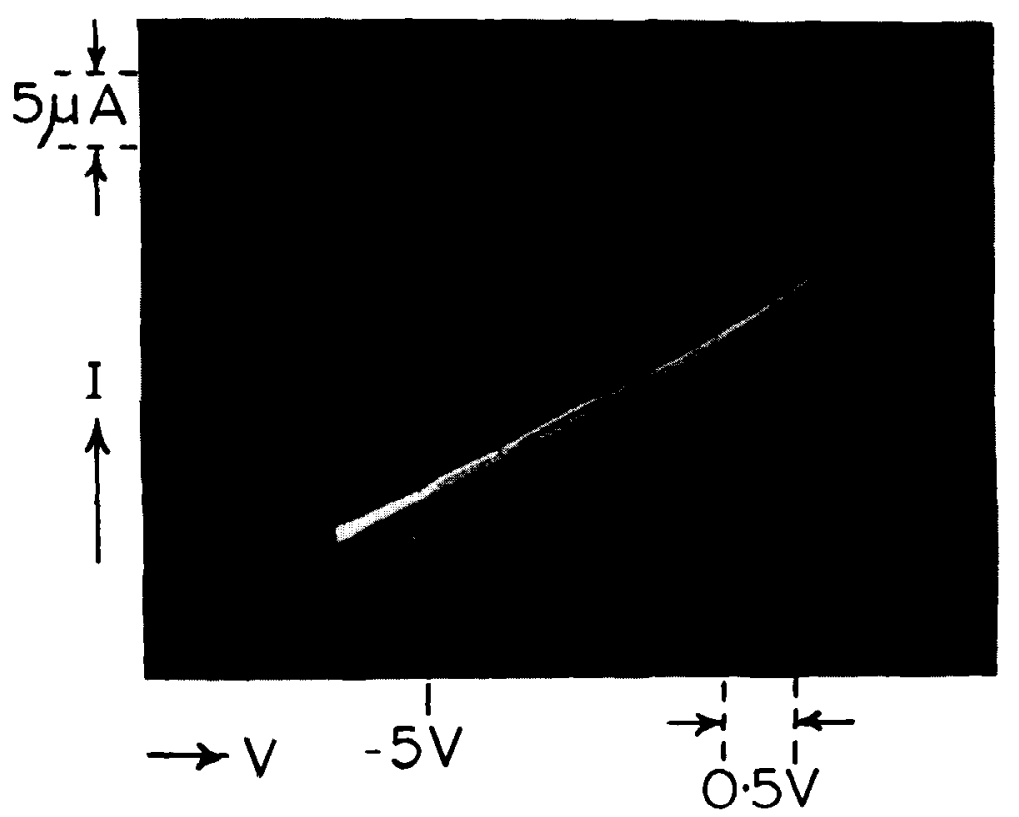

FIG. 4. A dynamic I-V characteristic at a temperature $T=77^{\circ} \mathrm{K}$ showing the oscillations in the photocurrent and hysteresis (sample no. 9).

${ }^{150 \mathrm{kcls}} W^{\mathrm{T}=85^{\circ} \mathrm{K}}$

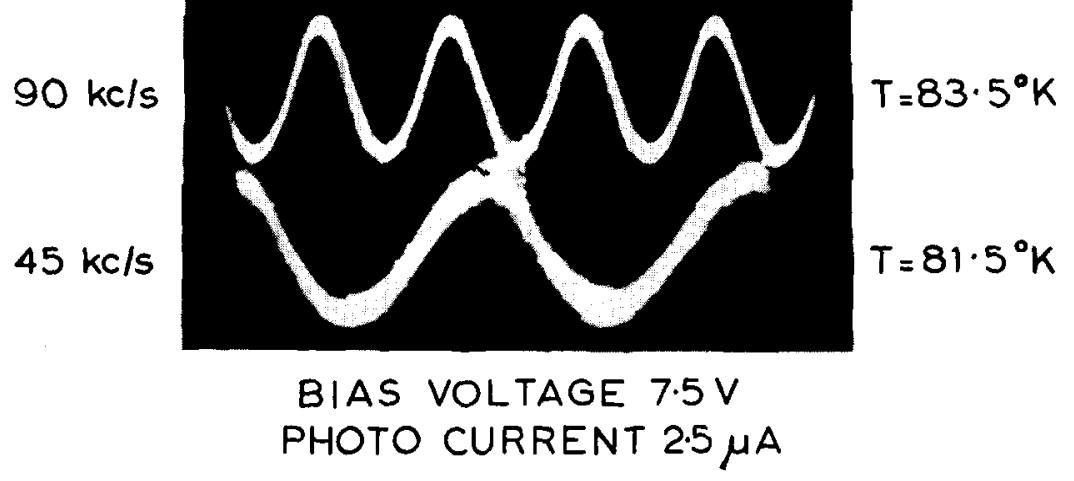

FIG. 9. The sinusoidal oscillations in the photocurrent at various temperatures.

[facing p. 84 


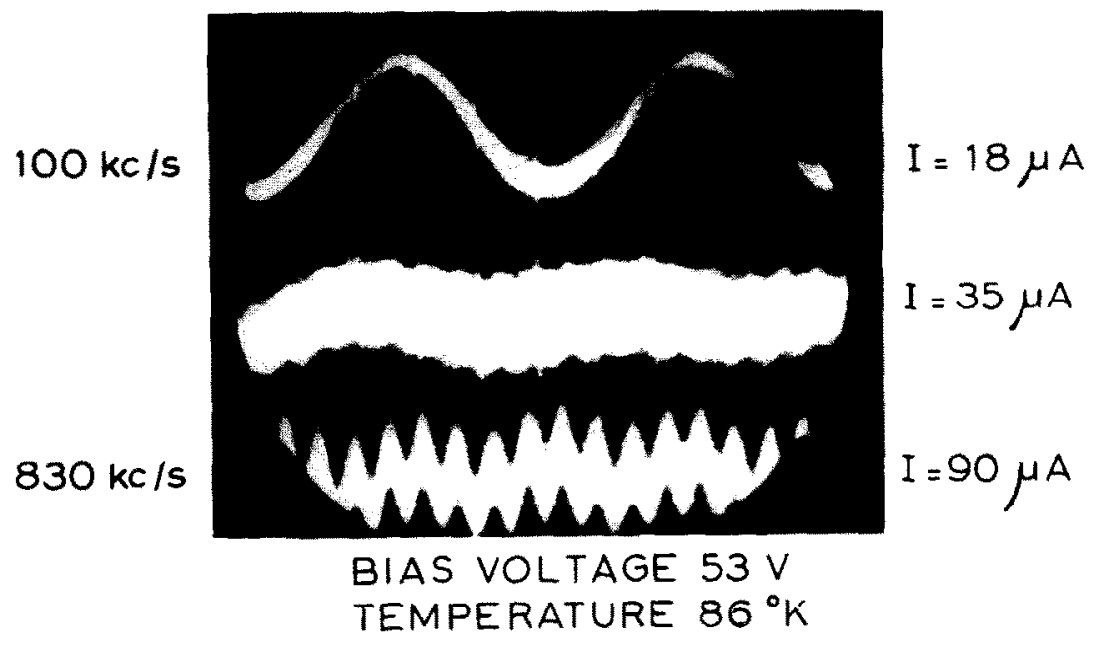

Fu; 12. The oscillation in the photocurrent at various illumination levels.

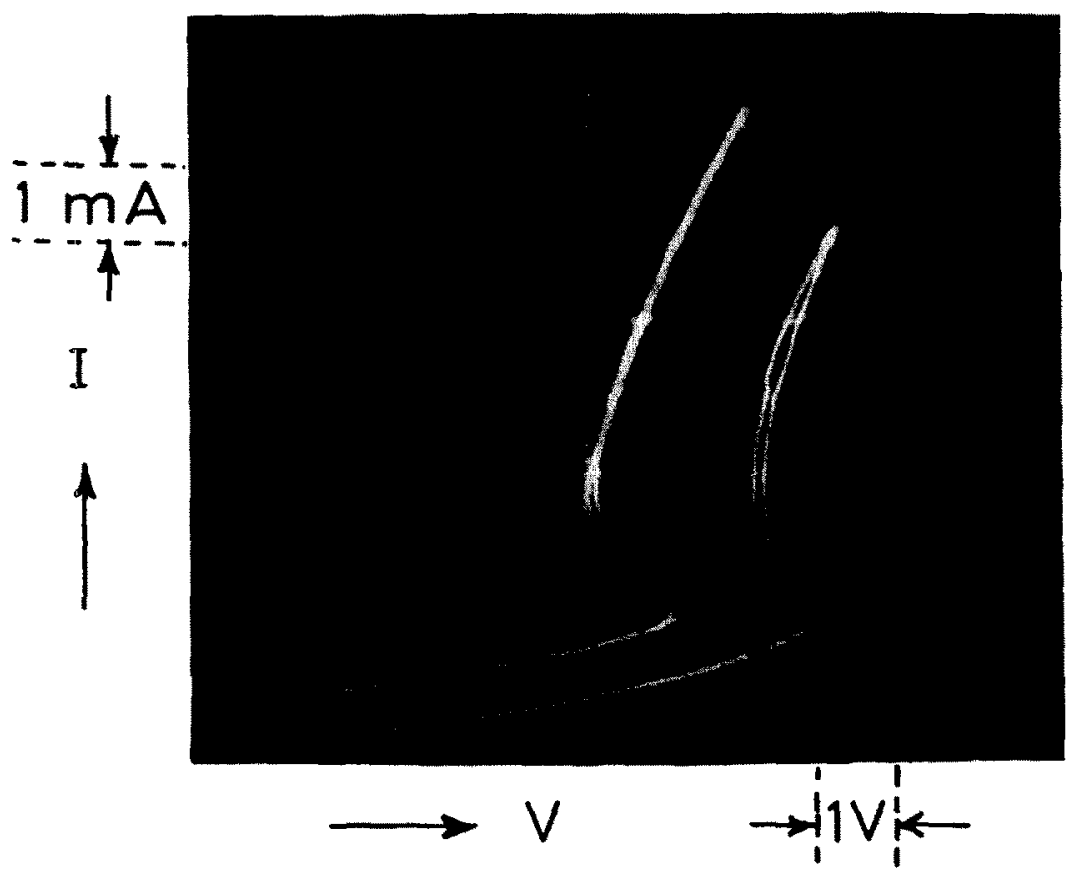

FIC. 18. Negative resistance region of the I-V characteristic at positive bias voltages with and without illumination (sample no. 1). 


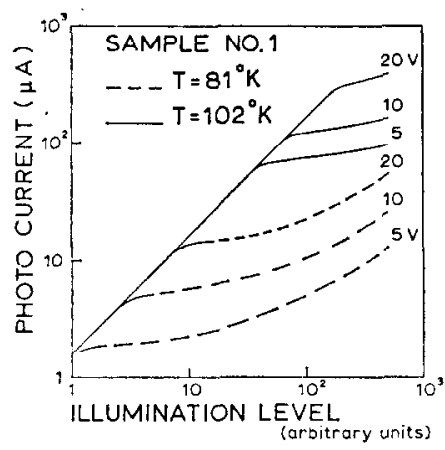

FIG. 5. The photocurrent as a function of illumination level for various voltages and at two temperatures.

illumination level was varied. We found that either lowering the temperature or increasing the illumination level affect the characteristics in a similar way. A dynamic current-voltage characteristic is shown in Fig. 4. Note the oscillations in the photocurrent and the hysteresis of the curve.

The photocurrent as a function of illumination level has been plotted in Fig. 5. The illumination level has been varied by means of attenuation

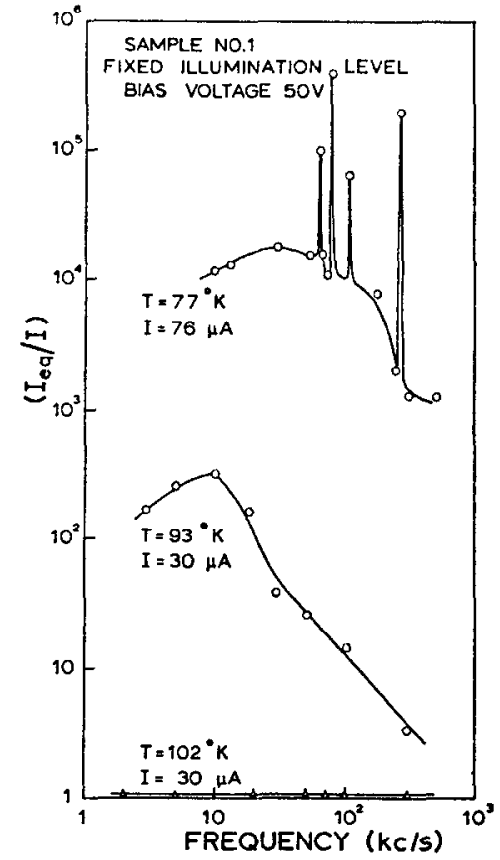

Fig. 6. Normalized noise density as a function of frequency at various temperatures. plates. The current first increases linearly with the illumination level, then nearly saturates, which means that it increases slightly with illumination level. At lower voltages the current saturates at lower illumination levels. These effects are more pronounced at lower temperatures.

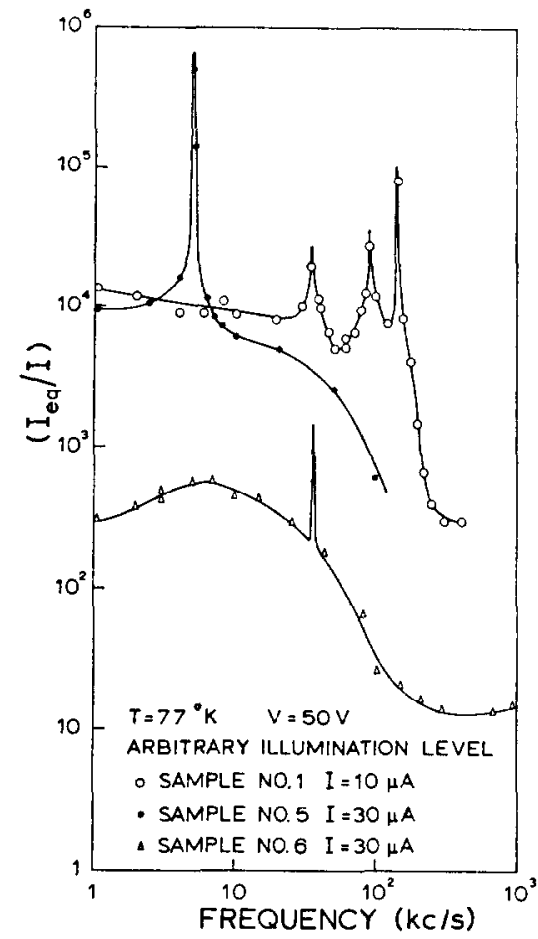

FrG. 7. Normalized noise density as a function of frequency for various samples.

(ii) Noise. The normalized spectral noise density at varying temperatures has been plotted in Fig. 6 at fixed illumination level and at fixed bias voltage. The noise density increases with decreasing temperature at all frequencies investigated. Some noise spectra of different samples are shown in Fig. 7. The cut-off frequency of the noise density is about $100 \mathrm{kc} / \mathrm{s}$. The noise spectra in Figs. 6 and 7 contain several peaks, which show up as sinusoidal oscillations on the oscilloscope screen. Their appearance makes a detailed study of the noise behaviour somewhat complicated. In general the noise density increases strongly with current and illumination level, with powers between 2 and 6 , and the noise density increases with voltage too. Breakdown 


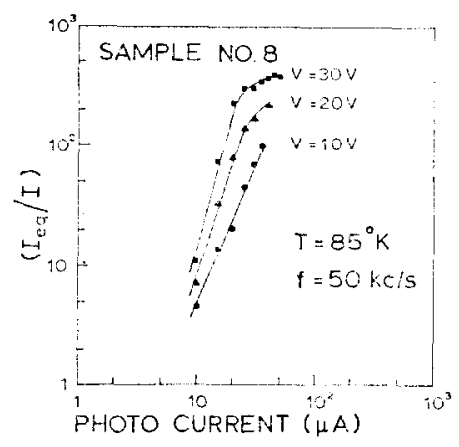

FIG. 8. Normalized spectral noise density as a function of photocurrent for various voltages.

phenomena occur at very high illumination levels, in particular when the voltage is near $100 \mathrm{~V}$ and the temperature is low $\left(\sim 77^{\circ} \mathrm{K}\right)$. The noise in the photocurrent of sample no. 8 could be investigated in more detail since in this sample no oscillations appear. Figure 8 shows the normalized spectral noise density as a function of current at different voltages. The current was varied by controlling the illumination level. The related $\mathrm{I}-\mathrm{V}$ characteristics are those shown in Fig. 5.

(iii) Oscillations. The frequency of the sinusoidal oscillation lies in the range from $1 \mathrm{kc} / \mathrm{s}$ to $1 \mathrm{Mc} / \mathrm{s}$.
The frequency is strongly dependent on the temperature, which is illustrated in Fig. 9. This photograph also shows the sinusoidal shape of the oscillation. Some measurements on frequency versus temperature have been plotted in Fig. 10 . From these curves two different activation energies have been determined. These are $\Delta E=0.25 \pm$ $0.03 \mathrm{eV}$ and $\Delta E=0.15 \pm 0.02 \mathrm{eV}$. The activation energy is not dependent on voltage or illumination level, unless surface effects are clearly present (see below). Sample no. 1 revealed an activation energy of about $0.25 \mathrm{eV}$ in the first stages of the experiment. After the diode had been etched, the activation energy was $0 \cdot 15 \mathrm{eV}$.

When the temperature was changed, the frequency of oscillation sometimes jumped to another value whereas the activation energy remained the same (see Fig. 10).

The frequency of oscillation increases with increasing illumination level as is shown in Fig. 11 for various fixed voltages and for two temperatures. The increase of frequency with illumination level is less at higher temperatures, whereas at temperatures near the critical temperature sometimes a small decrease is found. When the illumination level is changed an existing oscillation is sometimes replaced with an oscillation of a different

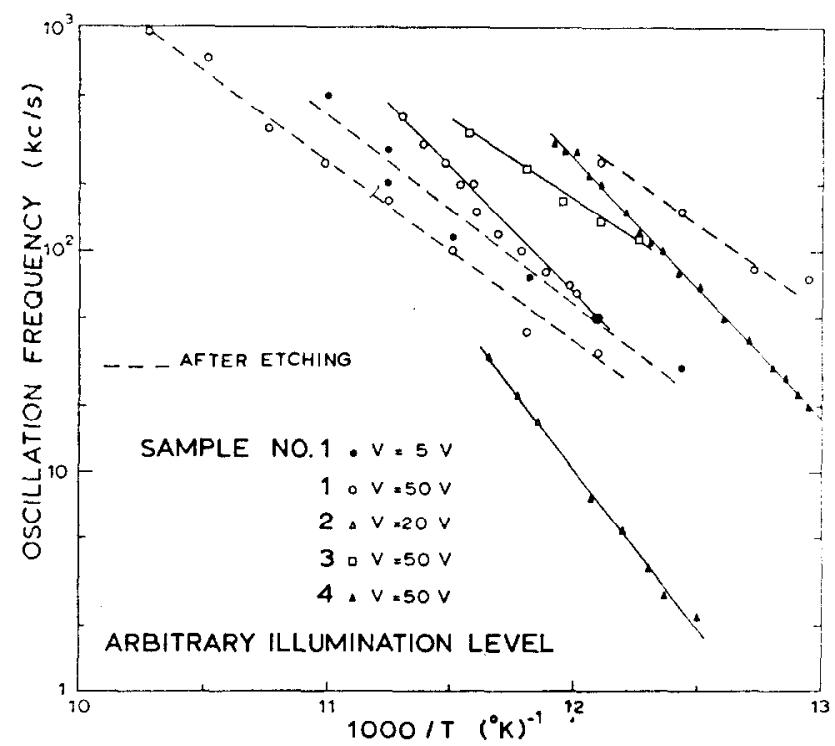

FIG. 10. The oscillation frequency as a function of temperature for various samples at arbitrary illumination levels. 


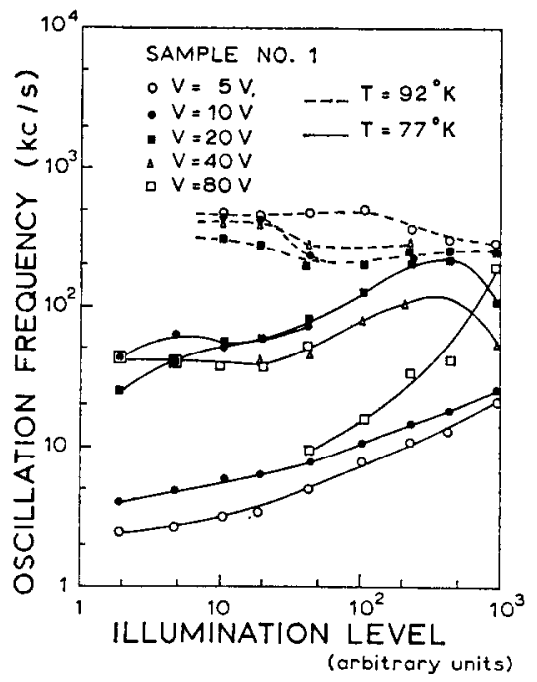

FIG. 11. The oscillation frequency as a function of illumination level for various voltages and at two temperatures.

frequency. This behaviour is also illustrated in Fig. 12, where the bias voltage and the temperature are fixed. These oscillations depend on the temperature as well as on the bias voltage in the same way. The oscillation frequency is approximately constant with changing bias voltage, an example of which is shown in Fig. 13. In this case the oscillations shown were present in the photocurrent simultaneously. Between 10 and $20 \mathrm{~V}$

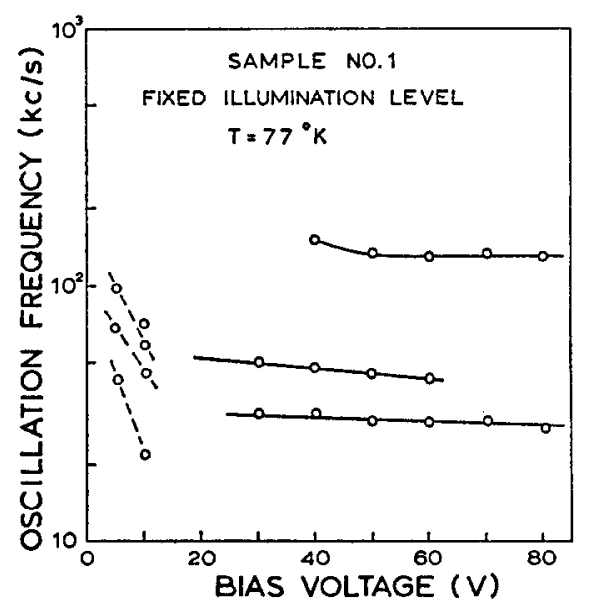

FIG. 13. The oscillation frequency as a function of bias voltage for fixed illumination level and at fixed temperature. there was no oscillation detectable, which was often the case in this voltage range at the lowest temperatures. Below $10 \mathrm{~V}$ the frequency of oscillation was observed to increase with decreasing voltage.

The frequency of oscillation was found to be independent of the wavelength of the light from 0.7 to $1.5 \mu$, leaving the photocurrent fixed. The shape of the oscillation is not always sinusoidal, especially so at higher illumination levels, in which cases higher harmonics may be detected too. The frequency as well as the shape of the oscillation is independent of external circuit parameters.

The amplitude of the oscillation is a more complicated function of the different parameters. We describe the general rules in the following.

The oscillation amplitude increases with increasing bias voltage as well as with increasing illumination level. With varying illumination level the oscillation amplitude behaves as $I^{\alpha}$, where $\alpha$ is between 2 and 3 for low illumination levels and $\alpha \sim 1$ for high illumination levels, independent of the bias voltage in first approximation. For voltages below $10 \mathrm{~V}$ the oscillation amplitude behaves as $V^{\alpha}$, where $\alpha$ is between 2 and 3 , the higher the illumination level, the larger is $\alpha$. Above $20 \mathrm{~V}$ the oscillation amplitude increases only slightly with increasing voltage.

The dependence on temperature of the oscillation amplitude may be described in general as increasing with decreasing temperature at low illumination levels and decreasing with decreasing temperature at very high illumination levels. The modulation depth of the photocurrent was always in the range of 0.01 to 1 per cent.

We did not find a specific relation between the appearance of the oscillation and the dependence of photocurrent on voltage or illumination level.

In one sample (no. 3) there existed an oscillation, whereas the noise remained near the shot noise level. The normalized spectral noise density is shown in Fig. 14 at arbitrary illumination level and at fixed voltage and temperature. There appears to be a very small increase of noise above the shot noise level. The frequency of oscillation was found to be slightly dependent on bias voltage and photocurrent in a way as described above. The frequency dependence on temperature reveals an activation energy equal to $\Delta E=0 \cdot 15 \pm 0.02 \mathrm{eV}$ (see Fig. 10). The form of the current-voltage 


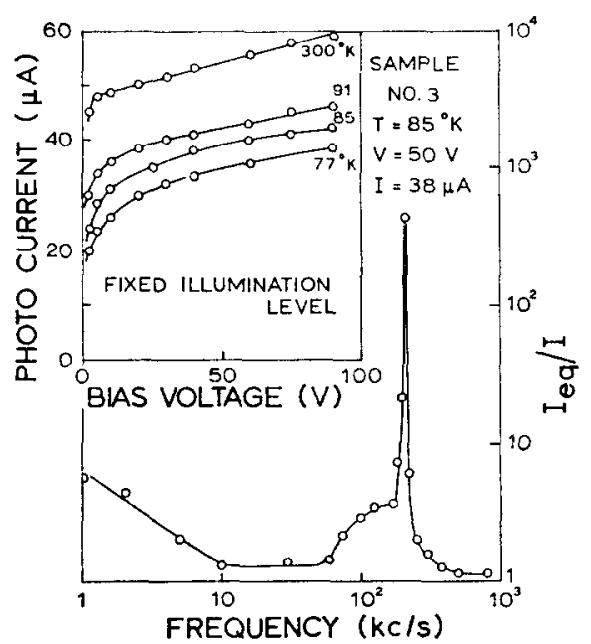

FIG. 14. The normalized noise density as a function of frequency and $\mathrm{I}-\mathrm{V}$ characteristics at various temperatures of sample no. 3 (see text).

characteristics remains almost unchanged when the temperature is lowered as is shown in the inset of Fig. 14.

(iv) Influence of ambient gas and surface treatment on the behaviour. We have attempted to determine the possible role of the ambient gas and surface treatment in the noise and oscillation effects described.

After removal of the glass window the noise and oscillations also appeared. In the ambient gases, nitrogen, oxygen and hydrogen, no variations due to the gases occurred. After chemical etching of the diode the effects sometimes increased, sometimes decreased. The current-voltage characteristics may have a form that is different from that shown in Figs. 3 and 4, c.g. currents incrcasing with the voltage to values that exceed the saturation current. This may be the case for instance, when wet air is let in. After the diode has been etched the curves are again of the type as shown in Figs. 3 and 4 . The behaviour of the noise before etching is comparable with that after etching. 'The dependence of oscillation frequency on voltage and illumination level is also similar before and after etching. The dependence of oscillation frequency on temperature was found to be sensitive to etching of the diode, if the $I-V$ characteristics were different from those in Figs. 3 and 4. These effects were not reproducible.
From these experiments we conclude that the surface treatment may influence the effects described, but that it is not a crucial factor in their origin.

\section{DISCUSSION}

(a) Evidence of the presence of gold impurity levels in the diode

The presence of gold in the diodes seemed to be an important factor in the effects described. We therefore measured the local photo-response curves and the temperature dependence of the dark current, which gave evidence to the presence of gold and from which the gold concentration could be estimated.

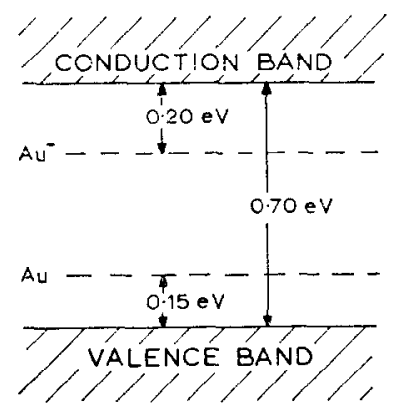

FIG. 15. Energy level scheme of gold-doped germanium.

The properties of gold-doped germanium have been described by many authors ${ }^{(26-28)}$ and it is well known that impurity centres of gold in germanium can have four energy levels in the forbidden band. Between 77 and $300^{\circ} \mathrm{K}$ we have to deal with two energy levels only (see Fig. 15). The lowest level is an acceptor level $0.15 \mathrm{eV}$ above the valence band, which is formed by ncutral gold atoms. The upper level is an acceptor level $0.20 \mathrm{eV}$ below the conduction band, which is formed by singly charged negative gold ions.

The $p$-region of our diode has been obtained by gold doping of $n$-type germanium. When carrier generation and recombination in the transition region, as given by SHOCKLEY and $\operatorname{READ}^{(29)}$ is taken into account, we will understand the observed reverse dark current dependence on temperature with the activation energy of about $0.50 \mathrm{eV} .^{(30)}$ Calculations show that this generation-recombination current is, indeed, large compared to the diffusion current at low temperatures. 
We may learn more about the concentration of gold atoms by comparing the lifetime of the electrons in the $p$-region, which can be determined from the photoresponse curves (see Fig. 1), with the lifetime which can be calculated from the Shockley-Read recombination theory. ${ }^{(29)}$ The diffusion length $L_{n}$ of the electrons in the $p$-region is determined from Fig. 1 to be about $5 \times 10^{-3} \mathrm{~cm}$ (we consider one dimensional transport only). By using $L_{n}=\left(\sqrt{ } D_{n} \tau_{n}\right)$ and $D_{n} \simeq 100 \mathrm{~cm}^{2} / \mathrm{sec}$ we calculate then: $\tau_{n} \simeq 2.5 \times 10^{-7}$ sec.

At room temperature most of the Au centres are occupied by electrons. The occupied Au centres form the empty $\mathrm{Au}^{-}$centres. When holeelectron pairs are generated by illumination from band to band, electrons are captured from the conduction band by the empty $\mathrm{Au}^{-}$centres, rather than holes from the valence band by the occupied Au centres, since $\sigma_{n}{ }^{-} \gg \sigma_{p}{ }^{0}{ }^{(31)}$ Then the occupied $\mathrm{Au}^{-}$centres capture holes from the valence band $\left(\sigma_{p}{ }^{-} \gg \sigma_{p}{ }^{0}\right)$. The electron-hole recombination therefore takes place mainly through the $\mathrm{Au}^{-}$centres. The Shockley-Read theory ${ }^{(29)}$ gives for the electron lifetime in the case of an arbitrary density of traps and not too high an illumination level ${ }^{(32)}$

$\tau_{n}=\frac{\tau_{p 0}\left(n_{0}+n_{1}\right)+\tau_{n_{0}}\left[p_{0}+p_{1}+N_{t}\left(1+p_{0} / p_{1}\right)^{-1}\right]}{n_{0}+p_{0}+N_{t}\left(1+p_{0} / p_{1}\right)^{-1}\left(1+p_{1} / p_{0}\right)^{-1}}$

where the usual symbols have been used, which have already been listed.

The lifetimes $\tau_{n_{0}} \propto\left(N_{t} \sigma_{n}{ }^{-} v\right)^{-1}$ and $\tau_{p_{0}}$ $\propto\left(N_{t} \sigma_{p}{ }^{-} v\right)^{-1}$ and since $\sigma_{n}{ }^{-} \ll \sigma_{p}{ }^{-}$is $\tau_{n_{0}} \gg \tau_{p_{0}}$. The Fermi level lies below the recombination level involved such, that $n_{0} \ll n_{1}$. Since $N_{t}>N_{d} \simeq 10^{14}$ $\mathrm{cm}^{-3 *}$ it is likely that $p_{0}>10^{14} \mathrm{~cm}^{-3}$ and with $p_{0} n_{0}=n_{i}^{2}$ where $n_{i} \simeq 2.4 \times 10^{13} \mathrm{~cm}^{-3}$ follows $n_{0} \ll p_{0}$. The expression for the lifetime $\tau_{n}$ may then be simplified in $\tau_{n} \simeq \tau_{n 0}\left(1+p_{1} / p_{0}\right)$. And since $p_{1} \simeq p_{0}$ becomes $\tau_{n} \simeq 2 \tau_{n_{0}}$, so that $\tau_{n} \simeq 2\left(N_{t} \sigma_{n}{ }^{-} v\right)^{-1}$. With $\sigma_{n}{ }^{-} v \simeq 2 \times 10^{-9} \mathrm{~cm}^{3}$ $\mathrm{sec}^{-1(33)}$ and $\tau_{n} \simeq 2.5 \times 10^{-7} \mathrm{sec}$ from the photoresponse curves we find $N_{t} \simeq 4 \times 10^{15} \mathrm{~cm}^{-3}$. The solubility of gold in germanium is limited to about $3 \times 10^{15} \mathrm{~cm}^{-3} \cdot{ }^{(26)}$ Our result indicates that a

* The resistivity of the $n$-material is $5-15 \Omega-\mathrm{cm}$. This means that $N_{d} \simeq 1-5 \times 10^{14} \mathrm{~cm}^{-3}$. concentration of this order of magnitude is present in the $p$-region of the diode.

From the foregoing discussion we have obtained evidence that the diodes are gold-doped indeed, and we have gained some information on the gold concentration.

\section{(b) Behaviour at temperatures below the critical temperature}

(i) Photocurrent. I-V characteristics as shown in Figs. 2 and 3 may be explained by assuming a large resistance in series with the junction, even under conditions of high illumination. This

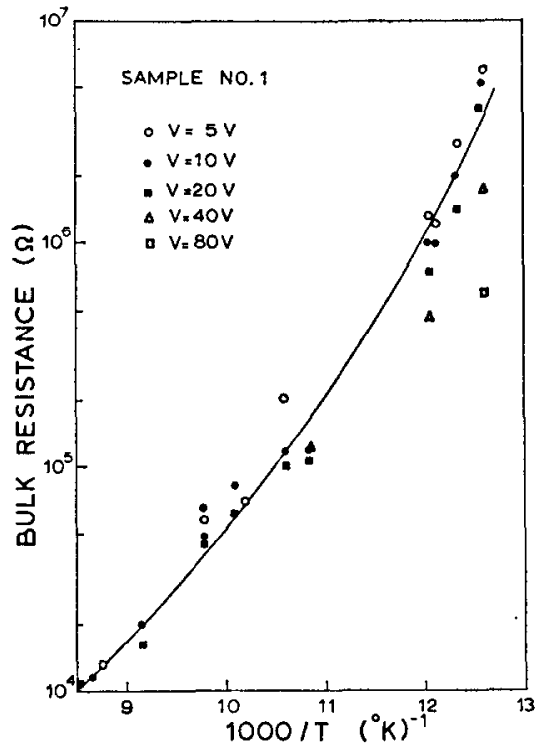

FIG. 16. Bulk resistance vs. temperature for various voltages.

resistance causes a large voltage drop, with the consequence that the junction voltage is lowered, or even becomes zero. In the latter case we expect a linear I-V characteristic with a slope determined by the value of the resistance. The increase of current at higher voltages (see Fig. 2) may be associated with surface effects, as mentioned already. From the current versus illumination level curves it is evident that such a large series resistance play a part, indeed. Figure 5 shows that the current saturates at a certain illumination level which is dependent on the bias voltage. From the "saturation point" in these curves we can calculate 
the value of the series resistance. Moreover, we can determine this resistance as a function of the temperature. The results obtained by assuming that Ohm's law holds at the "saturation point" are plotted in Fig. 16. In the temperature range below the critical temperature the order of magnitude of the resistance turns out to be $10^{5}-10^{7} \Omega$, increasing with decreasing temperature (we have corrected for the load resistance of $20 \mathrm{k} \Omega$ ). The activation energies which may be estimated from Fig. 16 lies between 0.10 and $0.20 \mathrm{eV}$, and increases with decreasing temperature. This behaviour is what we should expect if the series resistance is determined by the bulk of the $p$-region of the diodc. The steady state concentration of thermally generated free holes at low temperature is then given by

$$
p_{0} \simeq\left(N_{t}-N_{d}\right) \exp \left[\left(E_{F}-E_{t}\right) / k T\right],
$$

where $\left(E_{F}-E_{t}\right)$ is the energy difference between the Fermi level and the $0 \cdot 15 \mathrm{eV}$ gold acceptor level. Here optically generated holes have been neglected. The results plotted indicate that this is justified in first approximation. Note that in order to reach saturation a higher illumination level is required at higher voltages and that the resistance determined from this saturation is somewhat smaller at high voltages at the lowest temperatures only.

However, one would expect that optically generated holes contribute considerably to the conductivity of the $p$-region at the moderate illumination levels used. We will now show that the foregoing discussion, where we have neglected the optically generated holes remains still valid, if we take into account the different diffusion lengths of the minority carriers in the $p$ - and $n$-region.

We know that

$$
L_{p} / L_{n}=\left(D_{p} \tau_{p} / D_{n} \tau_{n}\right)^{1 / 2} .
$$

At $100^{\circ} \mathrm{K}$ is $D_{p} \simeq D_{n}$. Since the $p$-region is golddoped the lifetime of the electrons in the $p$-region will be much smaller than the lifetime of the holes in the $n$-region: $\tau_{n} \ll \tau_{p}$. So $L_{p} \gg L_{n}$.*

* 'The numerical values of $L_{p}$ and $L_{n}$ may be estimated. From Fig. $1 \tau_{p} \simeq 10^{-5} \mathrm{sec}$ at $300^{\circ} \mathrm{K}$. If we neglect a small temperature dependence of $\tau_{p}$ we find that $L_{p}\left(100^{\circ} \mathrm{K}\right) \simeq 0.5 \mathrm{~mm}$. A calculation of $\tau_{n}$ at low temperature is rather complicated, since we have to deal with a four-level system. With the estimate $\tau_{n} \leqslant 10^{-6}$ sec we find $L_{n}\left(100^{\circ} \mathrm{K}\right) \lesssim 0.15 \mathrm{~mm}$.

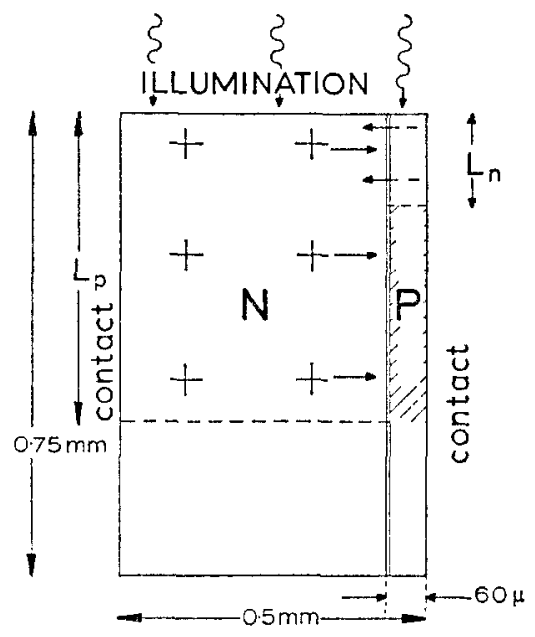

FIG. 17. A cross-section of the diode perpendicular to the $p-n$ junction approximately on scale for temperatures below the critical temperature. The shadowed $p$-region is called the "dark" p-region.

We can now easily understand the behaviour of the photocurrent described. A cross section of the diode perpendicular to the $p-n$ junction has been sketched approximately on scale in Fig. 17. A large portion of the photocurrent consists of holes which are injected from the $\boldsymbol{n}$-region into the highresistivity lower part, the "dark part" of the $p$-region. This current will saturate also if the

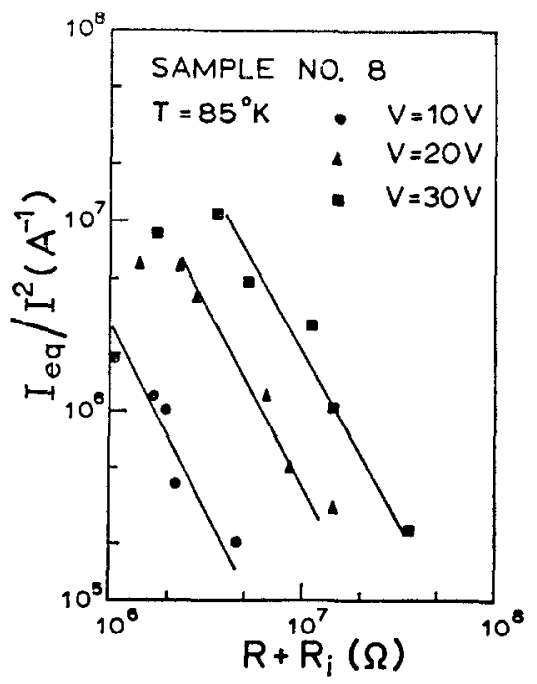

FIG. 19. $I_{e q} / I^{2}$ vs. $\left(R+R_{t}\right)$ for various voltages (see text). 
"light" part of the p-region has a low resistance. The observed small linear increase of photocurrent with illumination level after the saturation point may then be attributed to the contribution of the upper part of the diode. The assumption of the large series resistance was found to be supported also by the behaviour below the critical temperature at positive bias voltages. The $\mathrm{I}-\mathrm{V}$ characteristics exhibit after an ohmic part a region with a negative resistance (see Fig. 18), which may arise owing to double injection. ${ }^{(34,35)}$ In this negative resistance region of the characteristics we observed oscillations in the current which are dependent on the external circuit parameters.

(ii) Noise. The noise behaviour of the diode may also be understood when the large bulk resistance is taken into account. We will show this by means of the following model.* Consider a $p-n$ junction with a series resistance $R$, which show fluctuations $\Delta R$. Let $R$ be the resistance of the $p$-region, and let $V$ be the voltage across the junction. The voltage across the junction plus the series resistance is the fixed applied voltage.

The variation of current $I$ flowing through this device is

$$
\Delta I=\Delta I_{0}+(\mathrm{d} I / \mathrm{d} V) \Delta V,
$$

where $\Delta I_{0}$ is the current fluctuation, when the voltage across the transition region is constant, i.e. $\Delta I_{0}$ accounts for the shot noise. Assuming $R$ independent of $I$ we have

$$
\Delta V=-I \cdot \Delta R-R \cdot \Delta I .
$$

Substituting equation (6) into (5) yields

$$
\Delta I=\left(R_{i} \cdot \Delta I_{0}-I \cdot \Delta R\right) /\left(R_{i}+R\right),
$$

where $R_{1} \equiv \mathrm{d} V / \mathrm{d} r$.

Since $\Delta I_{0}$ and $\Delta R$ are independent we find for the mean square fluctuation

$$
\Delta I^{2}=\Delta I_{0}^{2} R_{i}^{2} /\left(R_{i}+R\right)^{2}+\Delta R^{2} I^{2} /\left(R_{i}+R\right)^{2} \text {. }
$$

The quantity $\Delta R^{2}$ represents the fluctuations in the resistance of the $p$-region caused by fluctuations in the number of free holes. We know:

$$
\Delta R^{2}=R^{2} \Delta P^{2} / P_{0}^{2},
$$

where $P_{0}$ is the average total number of free

\footnotetext{
* For this analysis we are indebted to Dr. R. J. J. Zijlstra of our Laboratory.
}

holes in the $p$-region. Making a Fourier-analysis we find from equations (8) and (9) the spectral density $S_{i}(f)$, of the current fluctuations

$$
\begin{aligned}
S_{i}(f)= & S_{i_{0}}\left[\left(R_{i} /\left(R_{i}+R\right)\right]^{2}\right. \\
& +S_{p}(f)\left(I^{2} / P_{0}^{2}\right)\left[R /\left(R_{i}+R\right)\right]^{2} .
\end{aligned}
$$

The spectral density $S_{p}(f)$ of the fluctuations $\Delta P$ in $P$ is ${ }^{(1)}$

$$
S_{p}(f)=4 \Delta P^{2} \tau_{p} /\left(1+\omega^{2} \tau_{p}{ }^{2}\right),
$$

where $\tau_{p}$ is the average lifetime of the holes. We have

$$
\begin{aligned}
S_{i}(f)= & S_{i_{0}}\left[R_{i} /\left(R_{i}+R\right)\right]^{2} \\
& +4 I^{2}\left(\Delta \boldsymbol{P}^{2} / P_{0}^{2}\right)\left[\tau_{p} /\left(1+\omega^{2}{\tau_{p}}^{2}\right)\right] \\
& \times\left[R /\left(R_{i}+R\right)\right]^{2} .
\end{aligned}
$$

Now we come to the calculation of $\Delta P^{2}$ and $\tau_{p}$. To this end we assume a single trapping level deep in the forbidden band, which is interacting with the valence band only. We also assume a shallow donor level, from which all electrons have been trapped. Then the calculation of $\Delta \boldsymbol{P}^{2}$ and $\tau_{p}$ can easily be performed, using a method developed by Burgess. ${ }^{(1,36)}$ Assuming that $p_{0} \ll N_{t}, N_{d}$ the result is

$$
\tau_{p} \simeq\left(N_{d} \sigma_{p} v\right)^{-1}
$$

and

$$
\Delta \boldsymbol{P}^{2}=g \tau_{p} \simeq P_{0},
$$

where $N_{d}$ is the concentration of donors $\sigma_{p}$ is the hole capture cross-section $v$ is the velocity of the holes

and $g$ is the generation rate of free holes.

The spectral density, $S_{1}(f)$, of the current fluctuations becomes then:

$$
\begin{aligned}
S_{i}(f)= & S_{i_{0}}\left[R_{i} /\left(R_{i}+R\right)\right]^{2} \\
& +\left[4 I^{2} / g\left(1+\omega^{2} \tau_{p}^{2}\right)\right]\left[R /\left(R_{i}+R\right)\right]^{2} .
\end{aligned}
$$

We note that for an ideal $p-n$ junction, in which there is a diffusion current only, $R_{t} \rightarrow \infty$ for reverse bias conditions and equation (15) reduces to $S_{i}(f)=S_{i_{0}}$. The spectral noise density then is equal to shot noise. In practice, however, the reverse current depends on the voltage making $R_{i}$ finite. By cooling the device $R$ increases and the 
contribution to $S_{i}(f)$ of the second term in equation (15) increases too.

Let in our case the $p-n$ junction and the dark $p$-region be represented by the described model. Then the hole lifetime $\tau_{p} \simeq\left(N_{d} \sigma_{p}^{0} v\right)^{-1}$, where $\sigma_{p}{ }^{0}$ is the cross-section for the capture of a hole by a singly ionized gold atom. Using $N_{d} \simeq 3 \times 10^{14} \mathrm{~cm}^{-3} \sigma_{p}{ }^{0} \simeq 3 \times 10^{-18} \mathrm{~cm}^{2(31)}$ and $v=v_{t h} \simeq 1.8 \times 10^{7} \mathrm{~cm} / \mathrm{sec}$ the hole lifetime at $80^{\circ} \mathrm{K}$ becomes $\tau_{p} \simeq 10^{-4}$ sec. Here $v_{t h}$ is the thermal velocity. The drift velocity of the holes in the $p$-region is calculated to be as large as $10^{8} \mathrm{~cm} / \mathrm{sec}$ at high voltages $(\sim 100 \mathrm{~V})$. Then $\tau_{p} \simeq 10^{-5} \mathrm{sec}$.

The value of the quantity $\Delta P^{2} \simeq P_{0}$ can be determined from

$$
P_{0}=l^{2} / e \mu_{p} R
$$

where $l$ is the length of the $p$-region, $\mu_{p}$ is the holc mobility and $R$ is the resistance of the dark p-region.

With $R \simeq 10^{5}-10^{6} \Omega, \mu_{p} \simeq 10^{4} \mathrm{~cm}^{2} / \mathrm{V}-\mathrm{sec}$ and $l \simeq 60 \mu$ we find $P_{0} \simeq 10^{3}-10^{4}$. The generation rate $g$ is then $g=10^{7}-10^{9}$.

From equation (15) we can show that this results in a high noise density. Assuming $R_{i} \lesssim R$ the ratio between the noise density and the shot noise is at low frequencies $\left(\omega \tau_{p} \ll 1\right)$ :

$$
S_{i}(0) / S_{i_{0}} \simeq I / e g,
$$

which may be as large as $10^{6}-10^{8}$.

Even when $R_{i} \gg R$, which is likely to be the case, the noise density may exceed shot noise level e.g. for $R_{t} \simeq 10^{7} \Omega, R \simeq 10^{5} \Omega$ and $I \simeq 10 \mu \mathrm{A}$ is $S_{i}(0) / S_{i_{0}} \simeq\left(R / R_{i}\right)^{2} I / e g \simeq 10^{2}$. Then it is clear why the noise density increases very strongly with decreasing temperature, since both $R$ and $g^{-1}$ increases exponentially with decreasing temperature with the activation energy of about $0 \cdot 15 \mathrm{eV}$.

The increase of noise density with increasing frequency which is often observed in a certain frequency range (see Figs. 6 and 7) can be explained by assuming that the differential resistance $Z_{i}$ of the junction is complex. When $Z_{i}$ consists of $R_{i}$ and $C_{i}$ parallel it can be shown easily that

$$
S_{i}(f) / S_{i_{0}} \simeq\left(R / R_{i}\right)^{2} I\left(1+4 \pi f^{2} C_{i}^{2} R_{i}^{2}\right) / e g,
$$

if $R \ll R_{i}$ and $2 \pi f C_{i} R_{i} \sim 1$.

Equation (18) shows that the noise density increases with frequency in the frequency range, where $2 \pi f C_{i} R_{i} \simeq 1$. With $R_{i} \simeq 10^{7} \Omega$ and $C_{i} \simeq 10-100 \mathrm{pF}$ we find $2 \pi f \simeq 10^{3}-10^{4} \mathrm{c} / \mathrm{s}$, which is the order of magnitude as observed.

The reciprocal hole lifetime should correspond to the cut-off frequency of the noise spectrum. The described model yields $\tau_{p} \simeq 10^{-4}-10^{-5} \mathrm{sec}$, whereas the measurements give $\tau_{p} \simeq 10^{-5} \mathrm{sec}$. The agreement is satisfactory when we realize that the model is much simpler than the reality. Factors, which may affect the noise behaviour are, for instance, transport processes and local space charges, ${ }^{(36)}$ contact properties and also the fact that there is a gradual transition from "dark" region to "light" region coupled with a gradual decrease of $R$ and $\tau_{p}$. Nevertheless, the model explains much of the observations. As mentioned before, a detailed study of the noise behaviour was difficult due to the presence of oscillations.

Fortunately there was one sample in which no oscillations occurred. Let us apply the model to the noise measurement of this sample, which has been plotted in Fig. 8. This shows the normalized noise density $I_{e q} / I$ vs. the photocurrent $I$, where the voltage was fixed and the photocurrent was varied via the illumination level. The deviation from the predicted quadratic dependence of the noise on the photocurrent may now be attributed to a decrease of $R_{i}$, assuming $R$ to be constant [see equation (15)]. This follows from Fig. 19, where we have plotted $I_{e q} / I^{2}$ against $\left(R_{i}+R\right)$. The quantity $\left(R_{i}+R\right)$ has been deduced from the $\mathrm{I}-\mathrm{V}$ characteristics of Fig. 3 , where it is simply equal to the slope of these curves. Figure 19 shows that in first approximation $I_{c q} / I^{2} \propto\left(R_{i}+R\right)^{-2}$, which supports our simple model. The increase of noise with increasing voltage might be related to an increase of $R$ owing to a drift effect.

The behaviour of sample no. 3 in which enhanced noise did not appear can be understood by assuming that the bulk resistance $R$ was too small compared with $R_{\mathfrak{i}}$ in the temperature range involved. This is in agreement with the $I-V$ characteristics, which also do not show up a large bulk resistance (see Fig. 14).

(iii) Oscillations. The dependence of the photocurrent on voltage and illumination level as well as on the noise behaviour of the diode are determined mainly by the high-resistivity "dark" $p$-region, as was shown already. We did not find any relation between the appearance of the 
oscillations and the macroscopic behaviour of the diode. Therefore we assume that the oscillations arise in the upper part of the diode. This assumption is supported by the observation that the frequency of the oscillation is dependent on the photocurrent when the current is varied via the illumination level (Fig. 11) and independent of the photocurrent, when the current is varied via the voltage for large voltages (Fig. 13). It should be borne in mind that in both cases the hole injection into the "dark" $p$-region varies, whereas the carrier density in the "light" $p$-region varies only by changing the illumination level and does not vary by changing the voltage. Note also the behaviour of sample no. 3 , in which an oscillation appears without the presence in the diode of a highresistivity "dark" $p$-region (see Fig. 14). The dependence of the frequency on voltage at the lowest voltages may be related to the finite resistance of the "light" p-region. This resistance is determined by the illumination level, and may be of the order of magnitude $10^{2}-10^{4} \Omega$. Variations in applied voltage in this voltage range cause a change in photocurrent as well as a change in voltage across the resistance.

The bchaviour of the frequency observed as a function of the applied voltage at the higher voltages should be considered carefully, since the voltage across the "light" $p$-region remains approximately constant. Therefore, if the "light" $p$-region is in an "oscillatory" state, it will be so in the whole range of applied voltages. The dynamic I-V characteristic of Fig. 4 shows clearly such a "bistable" state of the "light" p-region, though it would be more pronounced if the "dark" $p$-region did not predominate.

From Fig. 10 we know that in a given sample the temperature is the crucial parameter in determining the frequency of oscillation. One of the values of the activation energy found $(\Delta E=0.15 \mathrm{eV})$ indicates that the lowest acceptor level of gold sometimes plays an important part. This level controls the number of thermally generated holes in the valence band. The temperature determines the rate at which these holes are generated. The activation energy of $0.25 \mathrm{eV}$ might be due to a surface state or to other impurity centres $(\mathrm{Cu}$ for instance).

Such a temperature dependence of the oscillation frequency is expected in the so-called "recombination instability" described by BoNCHBRUEVICH and KaLASHNIKov ${ }^{(19,20)}$, and possibly observed by KuRova and Kalashnikov. ${ }^{(10)}$ These authors have shown that in a semiconductor, which contains two different types of impurity centres oscillations in the number of free carriers may arise. Onc type of impurity centres should have a capture rate, which increases with the energy of carriers. This property may result in a negative differential conductivity. The other type of impurity centres is assumed to tend to restore the system to a state of equilibrium. The rate of capture of electrons by negatively charged impurity centres increases with electric field, which was predicted by RIDLEY and WATKINS ${ }^{(37,38)}$ and confirmed experimentally for gold centres in $n$-type germanium at $20^{\circ} \mathrm{K} .{ }^{(11,33,39,40)}$ In our case the negatively charged $\mathrm{Au}^{-}$centres might play such a part. The other type of impurity centres assumed to be present for the appearance of the recombination instability ${ }^{(19,20)}$ then are the $\mathrm{Au}$ centres, giving the temperature dependence found. Since in our case both electrons and holes determine the conductivity of the "light" $p$-region, it is not an objection that the two types of centres are interacting with different bands.

Our actual situation is much more complicated than assumed in the derivation of the recombination instability. For instance, it is not easy to understand how the illumination and the presence of the junction affect the phenomena. Both are essential, since with forward bias voltage or without illumination these oscillations are not observed. Furthermore, there is in fact a gradual transition from "light" $p$-region (containing both free electrons and holes) to "dark" $p$-region (containing free holes only). In addition, we do not know the properties of the contacts. There are several oscillation phenomena, ${ }^{(15,18)}$ which were assumed to be associated with the properties of the contacts. In order to be more definite, it will be necessary to investigate better defined configurations.

Acknowledgements-We are very grateful to Professor Dr. C. Th. J. Alkemade for his advice during the course of this investigation, and for reading the manuscript. We are indebted to Dr. R. J. J. ZiJisTRA of this laboratory for several valuable discussions, in particular of the noise behaviour, and for reading the manuscript. One of us (P.T.B.) is indebted to Dr. M. T. VLAARDINGERBROEK 
of Philips' Research Laboratories, Eindhoven, for a useful discussion.

\section{REFERENCES}

1. A. v.D. ZiEL, Fluctuation Phenomena in Semiconductors, Butterworths, London (1959).

2. K. S. Champlin, J. appl. Phys. 30, 1039 (1959).

3. R. H. HAITZ, IEEE Trans. Electron Devices 12, 198 (1965)

4. A. S. TAger, Sov. Phys. solid St. 6, 1919 (1965).

5. F. J. Hyde, Proc. phys. Soc. 69B, 231 (1956).

6. M. Glicksman, Plasma Effects in Solids, 7th Int. Conf. Phys. of Semiconductors, Paris (1964) and references.

7. Special Issue on Semiconductor Bulk-Effect and Transit-time Devices, IEEE Trans. Electron Devices 13, January (1966).

8. M. C. Steele, K. ANdo and M. A. Lampert, J. phys. Soc. Japan 17, 1729 (1962).

9. N. Holonyak, Jr. and S. F. Bevacqua, Appl. Phys. Lett. 2, 71 (1963).

10. I. A. Kurova and S. G. Kalashnikov, Sor. Phys. solid St. 5, 2359 (1964).

11. B. K. Ridley and R. G. Pratt, J. Phys. Chem. Solids 26, 21 (1965).

12. S. M. Ryvkin, Proc. 7th Int. Conf. Phys. Semiconductors, Paris (1964).

13. H. Kroger, E. W. Prohofsky and H. R. Carleton, Phys. Rev. Lett. 12, 555 (1964).

14. S. Yamashita, E. Miyazaki and K. Miyaji, Jap. J. appl. Phys. 2, 661 (1963).

15. M. Cardona and W. Ruppel, J. appl. Phy's. 31, $1826(1960)$.

16. M. Krkuchi, J. phys. Soc. Japan 17, 240 (1962).

17. M. SAYER, Solid-St. Electron. 5, 409 (1962).

18. J. C. Guerci, B. Melchiorri and F. Melchiorri, Phys. Lett. 18, 243 (1965).

19. V. L. Bonch-Bruevich and S. G. Kalashnikov, Plasma effects in Solids, 7 th Int. Conf. Phys. Semiconductors, Paris (1964).

20. V. L. Bonch-Bruevich and S. G. KaLAshnikov, Sov. Phys. solid St. 7, 599 (1965).

21. H. C. J. van Burik and A. C. E. Wessels, Rev. scient. Instr. 34, 798 (1963).

22. F. S. Goucher, Phys. Rev. 81, 475 (1951).

23. A. R. F. Plummer, Proc. phys. Soc. B59, 539 (1956).

24. R. J. J. ZiJLSTRA (to be published).

25. R. J. J. Zijlstra, Thesis, Utrecht (1961).

26. W. C. Dunlap, Jr., Phys. Rev. 97, 614 (1955).

27. W. C. Dunlap, Jr., Phys. Rev. 100, 1629 (1955).

28. L. Johnson and H. Levinstein, Phys. Rev. 117, 1141 (1960).

29. W. Shockley and J. T. Read, Jr., Phys. Rev. 87, 835 (1952).

30. E. M. Pell, J. appl. Phys. 26, 658 (1955).

31. I. V. Karpova, V. G. Alekseeva and S. G. Kalashnikov, Sov. Phys. solid St. 4, 461 (1962) and references.

32. J. S. Blakemore, Semiconductor Statistics, Pergamon Press, Oxford (1962).
33. R. G. Pratt and B. K. Ridley, Proc. phys. Soc. 81, 996 (1963).

34. M. A. Lampert, Rep. Progr. Phys. 27, 329 (1964).

35. V. I. Stafeev, Sov. Phy's. solid St. 5, 2267 (1964).

36. K. M. VAN VIIET and J. R. FASSETT, Fluctuation Phenomena in Solids (Ed. R. E. BuRGEss) p. 267 , Academic Press, New York (1965).

37. B. K. Ridley and T. B. Watkins, Proc. phys. Soc. 78, 293 (1961).

38. B. K. Ridley and T. B. Watkins, J. Phys. Chem. Solids 22, 155 (1961).

39. B. K. Ridley and R. C. Pritt, Phys. Lett. 4, 300 (1963).

40. V. P. Sondaevskit, E. I. Karakushan and V. I. Stafeev, Phys. Stat. Sol. 14, 241 (1966).

41. R. H. Kingston, J. appl. Phys. 27, 101 (1956).

42. R. Bray and R. W. Cinningham, J. Phys, Chem. Solids 8, 99 (1959).

\section{APPENDIX}

\section{Quenching of the oscillations}

In one sample (no. 4) we found effects, which might inform us more about the origin of the described phenomena. Sample no. 4 exhibited an increase of noise and oscillations in the photocurrent, just as described before. The dependence of the oscillation frequency on temperature is plotted in Fig. 10. If the sample was irradiated with u.v. radiation in addition to the illumination, which gives the noise and oscillations, then the oscillations are quenched. The quenching is faster as the intensity of the u.v. radiation is higher. Quenchtimes are from less than $0.1 \mathrm{sec}$ up to several minutes. The active wavelength of the $u . v$. radiation is from 0.34 to $0.40 \mu$, with a maximum at $0.37 \mu$. After quenching the noise density is decreased somewhat, but it still is much ahove the shot noise level. The only way to get again the noise and oscillation is to heat the sample up to at least $20^{\circ} \mathrm{C}$, and to lower the temperature again below the critical temperature. Heating temperatures of 30 and $40^{\circ} \mathrm{C}$ results after cooling in more pronounced oscillations.

The dark current at room temperature was about one order of magnitude larger than the normal dark current and increased with voltage. The dependence on temperature yielded an activation energy of about $0.55 \mathrm{eV}$ at voltages below $1 \mathrm{~V}$. The decrease of activation energy with increasing voltage was stronger than that of other samples.

The photocurrent was independent of temperature from room temperature down to $170^{\circ} \mathrm{K}$, after which it decreases. Ultra-violet radiation did not affect the photocurrent above $170^{\circ} \mathrm{K}$. Below this temperature u.v. radiation did increase the photocurrent to the value above the temperature within a few minutes. The photocurrent does not change again after blocking the u.v. radiation.

The dark current was also affected by the u.v. radiation, but in a rather complicated way.

It is evident that surface effects are responsible for these phenomena. It is known ${ }^{(41)}$ that at a germanium 
surface an oxide layer and slow adsorption states may be present. BRAY and CUNNINGHAM ${ }^{(42)}$ have made surface studies on gold-doped germanium at $85^{\circ} \mathrm{K}$. They found a strong surface photoconductivity with radiation between 0.32 and $0.45 \mu$ wavelength, peaking near $0.37 \mu$, which they associated with the slow adsorption states outside the oxide layer.

We note that this wavelength range is just the quenching radiation in our case. Electrons generated by this radiation may pass the oxide barrier and may be trapped in the outer adsorption states. Then a compensating space charge layer is formed by free holes. From the observed quenching of the oscillation we may assume that the condition required for the occurrence of the oscillation is not fulfilled then. When the sample is heated the trapped electrons become free, the accumulation layer is removed and the oscillations can appear again. The observations on this sample are again an indication that the oscillations take place in the upper part of the diode. 